\title{
Correction to: Complex multiple risk intervention to promote healthy behaviours in people between 45 to 75 years attended in primary health care (EIRA study): study protocol for a hybrid trial
}

\author{
Edurne Zabaleta-del-Olmo 1,2,3,4*, Haizea Pombo ${ }^{5}$, Mariona Pons-Vigués ${ }^{1,3,4}$, Marc Casajuana-Closas ${ }^{1,3}$, \\ Enriqueta Pujol-Ribera ${ }^{1,2,3,4}$, Tomás López-Jiménez ${ }^{1,3}$, Carmen Cabezas-Peña ${ }^{6}$, Carme Martín-Borràs ${ }^{7,8}$, \\ Antoni Serrano-Blanco ${ }^{9,10}$, Maria Rubio-Valera ${ }^{9,10}$, Joan Llobera ${ }^{11}$, Alfonso Leiva ${ }^{11}$, Caterina Vicens ${ }^{11}$, Clara Vidal ${ }^{11}$, \\ Manuel Campiñez ${ }^{12}$, Remedios Martín-Álvarez ${ }^{12}$, José-Ángel Maderuelo ${ }^{13}$, José-Ignacio Recio ${ }^{13,14}$, \\ Luis García-Ortiz ${ }^{13,15}$, Emma Motrico ${ }^{16}$, Juan-Ángel Bellón ${ }^{17,18,19,20}$, Patricia Moreno-Peral ${ }^{17,18}$, \\ Carlos Martín-Cantera ${ }^{1,3}$, Ana Clavería ${ }^{21}$, Susana Aldecoa-Landesa ${ }^{21,22}$, Rosa Magallón-Botaya ${ }^{23}$ \\ and Bonaventura Bolíbar ${ }^{1,3}$
}

\section{Correction}

It has been highlighted the original article [1] contained a typesetting mistake in the authorship, and that author Caterina Vicens was omitted. This Correction article states the correct authorship. The original article has been updated.

It was later also highlighted that the below funding information was missing in the 'Funding' section: Maria Rubio-Valera has a contract awarded by the Pla Estratègic de Recerca i Innovació en Salut (PERIS) (Strategic Plan for Health Research and Innovation) by the Ministry of Health (Government of Catalonia) (SLT002/16/00190). This addition is not mentioned in the original article.

\section{Author details \\ 'Institut Universitarid'Investigació en AtencióPrimària Jordi Gol (IDIAP Jordi Gol), Gran Via Corts Catalanes 587 àtic, 08007 Barcelona, Spain. ${ }^{2}$ Gerència Territorial de Barcelona, Institut Català de la Salut, c/Balmes 22, 08007 Barcelona, Spain. ${ }^{3}$ Universitat Autònoma de Barcelona, Bellaterra, Cerdanyola del Vallès, Spain. ${ }^{4}$ Faculty of Nursing, Universitat de Girona, Carrer d'Emili Grahit, 77, 17003 Girona, Spain. ${ }^{5}$ Primary Care Research Unit of Bizkaia, Basque Health Service-Osakidetza, Luis Power Kalea 18, 48014 Bilbao, Spain. ${ }^{6}$ Deputy Directorate of Health Promotion, Public Health Agency, Department of Health Goverment of Catalonia, Roc Boronat, 81-95 (Edifici Salvany), 08005 Barcelona,}

\footnotetext{
* Correspondence: ezabaleta@idiapjgol.org

'Institut Universitarid'Investigació en AtencióPrimària Jordi Gol (IDIAP Jordi Gol), Gran Via Corts Catalanes 587 àtic, 08007 Barcelona, Spain

${ }^{2}$ Gerència Territorial de Barcelona, Institut Català de la Salut, c/Balmes 22, 08007 Barcelona, Spain

Full list of author information is available at the end of the article
}

Spain. ${ }^{7}$ Faculty of Psychology, Education and Sport Sciences (FPCEE) Blanquerna, Ramon Llull University, C/Císter 34, 08022 Barcelona, Spain. ${ }^{8}$ Faculty of Health Sciences (FCS) Blanquerna, Ramon Llull Univesity, C/ Padilla 326-332, 08025 Barcelona, Spain. ${ }^{9}$ Parc SanitariSant Joan de Déu, Institut de Recerca Sant Joan de Déu, C/Santa Rosa 39-57, 08950 Esplugues de Llobregat, Spain.

${ }^{10}$ Centro de Investigación Biomédica en Red de Epidemiología y Salud Pública (CIBERESP), Madrid, Spain. ${ }^{11}$ Gerènciad'AtencióPrimària de Mallorca, Institut de InvestigacióSanitària de les Illes Balears IdlSBa, C/Escola Graduada 3, 07002 Palma, Mallorca, Spain. ${ }^{12}$ Primary Health Centre Vallcarca, Edificio Pedraforca, Av. Vallcarca 169-205, 08023 Barcelona, Spain. ${ }^{13}$ Institute of Biomedical Research of Salamanca (IBSAL), Primary Health Care Research Unit, La Alamedilla Health Center, Health Service of Castilla y León (SACyL), Avda. Comuneros 27-31, 37003 Salamanca, Spain. ${ }^{14}$ Department of Nursing and Physiotherapy, University of Salamanca, Salamanca, Spain. ${ }^{15}$ Department of Biomedical and Diagnostic Sciences, University of Salamanca, Salamanca, Spain. ${ }^{16}$ Psychology Department, Universidad Loyola Andalucía, c/Energía Solar 1, Sevilla, Spain. ${ }^{17}$ Research Unit, Primary Care District of Málaga-Guadalhorce, c/ Sevilla, 23 Málaga, Spain.

${ }^{18}$ Institute of Biomedical Research in Málaga (IBIMA), c/ Sevilla, 23 Málaga, Spain. ${ }^{19} \mathrm{El}$ Palo Health Center, Andalusian Health Service (SAS), Av. Salvador Allende 159, 29018 Málaga, Spain. ${ }^{20}$ Department of Public Health and Psychiatry, University of Malaga, Campus de Teatinos s/n, 29071 Málaga, Spain. ${ }^{21}$ Grupo I-Saúde, Instituto de Investigación Sanitaria Galicia-Sur (IISGS), Xerencia de Xestión Integrada de Vigo, ServizoGalego de Saúde (SERGAS), Universidade de Vigo, Avda Rosalía Castro 21, 36201 Vigo, Spain. ${ }^{22}$ Primary Health Centre Beiramar, Xerencia de Xestión Integrada Vigo, Servizo Galego de Saúde (SERGAS), Avda Rosalía Castro 21, 36201 Vigo, Spain. ${ }^{23}$ Instituto de Investigación Sanitaria Aragón, Avda. San Juan Bosco 13, 50009 Zaragoza, Spain.

Received: 26 July 2018 Accepted: 26 July 2018

Published online: 13 August 2018

\section{Reference}

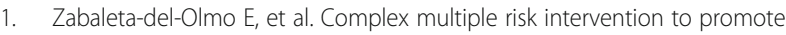
healthy behaviours in people between 45 to 75 years attended in primary health care (EIRA study): study protocol for a hybrid trial. BMC Public Health 2018;18:874. https://doi.org/10.1186/s12889-018-5805-y 